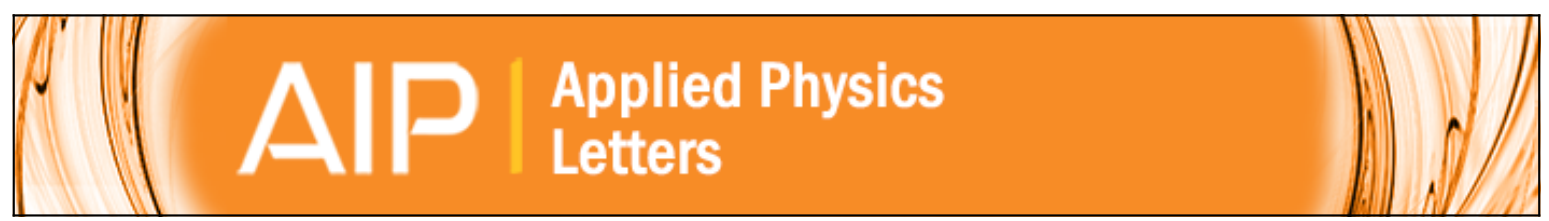

\title{
Influence of pulse duration, energy, and focusing on laser-assisted water condensation
}

Y. Petit, S. Henin, J. Kasparian, J. P. Wolf, P. Rohwetter, K. Stelmaszczyk, Z. Q. Hao, W. M. Nakaema, L. Wöste, A. Vogel, T. Pohl, and K. Weber

Citation: Applied Physics Letters 98, 041105 (2011); doi: 10.1063/1.3546172

View online: http://dx.doi.org/10.1063/1.3546172

View Table of Contents: http://scitation.aip.org/content/aip/journal/apl/98/4?ver=pdfcov

Published by the AIP Publishing

\section{Articles you may be interested in}

Cooperative effect of ultraviolet and near-infrared beams in laser-induced condensation

Appl. Phys. Lett. 103, 264103 (2013); 10.1063/1.4857895

Enhancement of anthracene fragmentation by circularly polarized intense femtosecond laser pulse

J. Chem. Phys. 126, 104304 (2007); 10.1063/1.2713107

Systematic study of resonance and off-energy-shell effects in laser-assisted electron scattering AIP Conf. Proc. 525, 583 (2000); 10.1063/1.1291975

$\mathrm{H} 2+$ in strong laser fields_-resolution of photofragments from different vibrational levels AIP Conf. Proc. 525, 502 (2000); 10.1063/1.1291967

Photoionization studies of small biological molecules using femtosecond laser pulses AIP Conf. Proc. 454, 206 (1998); 10.1063/1.57152

\section{AIP $\mid$ APL Photonics}

APL Photonics is pleased to announce Benjamin Eggleton as its Editor-in-Chief

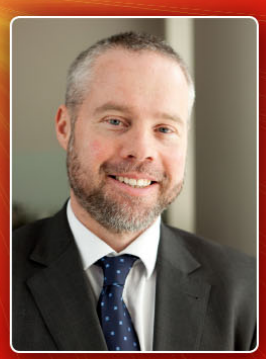




\title{
Influence of pulse duration, energy, and focusing on laser-assisted water condensation
}

\author{
Y. Petit, ${ }^{1}$ S. Henin, ${ }^{1}$ J. Kasparian, ${ }^{1, a)}$ J. P. Wolf, ${ }^{1}$ P. Rohwetter, ${ }^{2}$ K. Stelmaszczyk, ${ }^{2}$ \\ Z. Q. Hao, ${ }^{2}$ W. M. Nakaema, ${ }^{2}$ L. Wöste, ${ }^{2}$ A. Vogel, ${ }^{3}$ T. Pohl, $^{3}$ and K. Weber ${ }^{3}$ \\ ${ }^{1}$ Teramobile, GAP, Université de Genève, 20 rue de l'Ecole de Médecine, CH-1211 Genève 4, Switzerland \\ ${ }^{2}$ Teramobile, Institut für Experimentalphysik, Freie Universität Berlin, Arnimallee 14, D-14195 \\ Berlin, Germany \\ ${ }^{3}$ Department of Mechanical and Process Engineering, Environmental Measurement Techniques, \\ University of Applied Sciences, Düsseldorf, Josef-Gockeln-Str. 9, D-40474 Dusseldorf, Germany
}

(Received 15 November 2010; accepted 5 January 2011; published online 26 January 2011)

\begin{abstract}
We investigate the influence of laser parameters on laser-assisted water condensation in the atmosphere. Pulse energy is the most critical parameter. Nanoparticle generation depends linearly on energy beyond the filamentation threshold. Shorter pulses are more efficient than longer ones with saturation at $\sim 1.5$ ps. Multifilamenting beams appear more efficient than strongly focused ones in triggering the condensation and growth of submicronic particles, while polarization has a negligible influence on the process. The data suggest that the initiation of laser-assisted condensation relies on the photodissociation of the air molecules rather than on their photoionization. (C) 2011 American Institute of Physics. [doi:10.1063/1.3546172]
\end{abstract}

Self-guided filaments ${ }^{1-5}$ generated by ultrashort laser pulses have recently been proposed as candidates to assist the condensation of water in the atmosphere ${ }^{6}$ as an alternative to cloud seeding by dispersing small particles of carbonic ice, AgI, or salts. ${ }^{7}$ The mechanism behind laserassisted condensation most probably relies on photochemically activated processes, implying binary $\mathrm{H}_{2} \mathrm{O}-\mathrm{HNO}_{3}$ nucleation. Indeed, it was recently discovered that $\mathrm{O}_{3}, \mathrm{NO}$, and $\mathrm{NO}_{2}$ are generated in laser filaments in amounts largely sufficient to produce $\mathrm{HNO}_{3}$ in the multi-ppm range. ${ }^{8}$ Besides unveiling the mechanism of the phenomenon, the influence of the laser parameters is a key question for maximizing it and thus for obtaining macroscopic effects in the atmosphere.

In this work, we investigate in situ a wide range of laser energies $(0-165 \mathrm{~mJ})$, pulse durations (240 fs-3.3 ps), polarizations (linear and circular), and beam geometry (strong and loose focusing) and characterize their influence on filamentinduced water condensation in the atmosphere. We show that pulse energy, and to a lesser extent pulse duration, strongly affects the particle yield. In contrast, the impact of focusing is moderate, while the polarization of the incident beam has little influence on the particle generation efficiency. The different dependences of laser-induced condensation and ionization suggest that the activation of laser-assisted condensation relies on the photodissociation of the air molecules rather than their photoionization.

Experiments were performed under atmospheric conditions of $8-12{ }^{\circ} \mathrm{C}$ and $66 \%-80 \%$ relative humidity on the shore of the Rhône River close to Geneva $\left(46^{\circ} 12^{\prime}\right.$ North, $6^{\circ} 5^{\prime}$ East, and $380 \mathrm{~m}$ above sea level). This location was chosen to take benefit of the relatively warm water flow from the Lake of Geneva acting as a heat buffer, resulting in a local humidity enhancement.

Water vapor condensation was initiated by the Teramobile mobile femtosecond-terawatt laser system, ${ }^{9}$ providing up

${ }^{\text {a)} E l e c t r o n i c ~ m a i l: ~ j e r o m e . k a s p a r i a n @ u n i g e . c h . ~}$ to $165 \mathrm{~mJ}$ pulses of $240 \mathrm{fs}$ duration at a central wavelength of $800 \mathrm{~nm}$ and a repetition rate of $10 \mathrm{~Hz}$. The beam was expanded to a $10 \mathrm{~cm}$ diameter and slightly focused by a built-in expanding telescope. After $\sim 15 \mathrm{~m}$ of horizontal propagation $1.2 \mathrm{~m}$ above ground, the beam generated about 10 filaments in the atmosphere at the nonlinear focus. Alternatively, the beam was strongly focused on the measurement region using a $f=1 \mathrm{~m}$ lens.

Energy was attenuated by a half-wave plate associated with a polarizer inserted at the output of the main amplifier ahead of the compressor. The polarization was switched from linear to circular by a quarter-wave plate installed at the compressor exit. Furthermore, the pulse duration was adjusted by varying the distance between the gratings of the laser compressor so as to impose a linear chirp on the laser pulses, as described earlier. ${ }^{9}$

The measurements focused on the most intense filamenting region, which was shielded from external wind by an open protection chamber. The efficiency of the laser in aerosol generation was characterized by measuring the aerosol size distribution and number density with an aerosol spectrometer (Grimm 1.107, Grimm Aerosol Technik GmbH \& Co., Germany) sampling at a $2 \mathrm{~cm}$ distance from the laser filaments. This device measured the size distribution in 31 classes from $250 \mathrm{~nm}$ to $32 \mu \mathrm{m}$ diameter. It was coupled with a nanoparticle sensor (Grimm Nanocheck 1.320, Grimm Aerosol Technik GmbH \& Co., Germany). Alternating measurements in the filament-free atmosphere provided the particle background number density as a reference. Cross-check measurements using a condensation particle counter (TSI Model 3007, TSI Incorporated, Minnesota) allowed us to ensure that this measurement is free from artifacts due, e.g., to the electric charge deposed by the filaments on the particles. ${ }^{10}$ Simultaneously, the measurement of the shockwave by a sonometric setup described in detail before provided an estimation of the ionization of the air. ${ }^{11}$ When relevant, the statistical significance of the difference between experimental conditions was assessed by performing a Stu- 

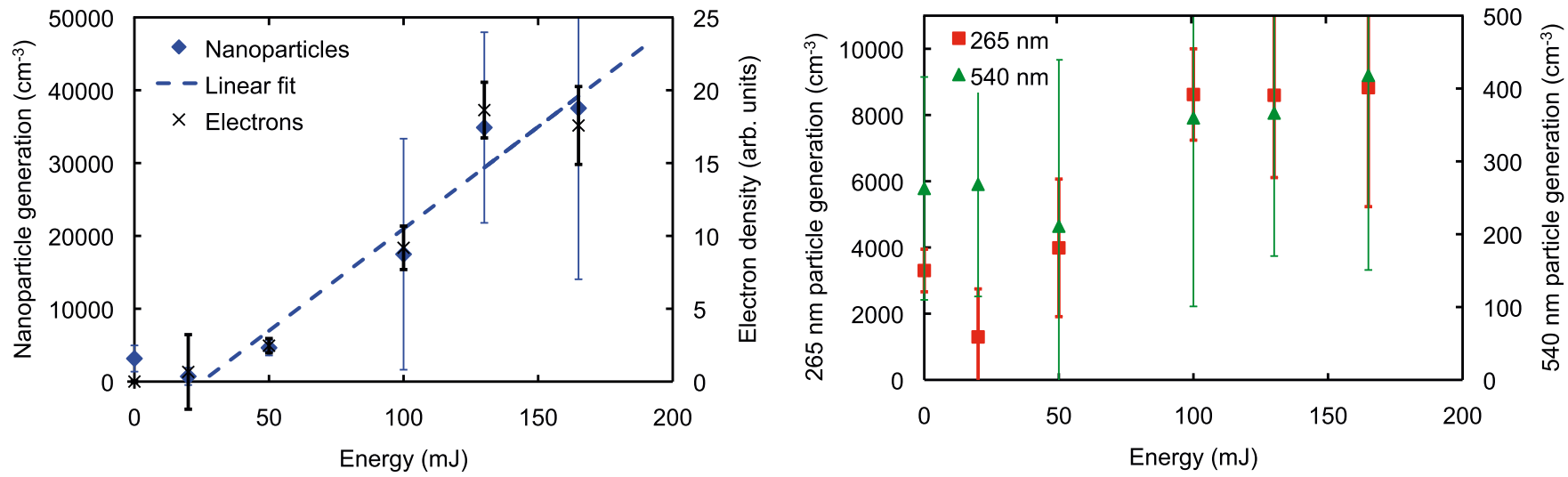

FIG. 1. (Color online) Energy dependence of the generation of (a) nanometric $(25 \mathrm{~nm})$ and (b) submicrometric particles by laser pulses of $240 \mathrm{fs}$ duration. Atmospheric conditions are $80 \%$ relative humidity and $12{ }^{\circ} \mathrm{C}$. Error bars display the standard deviation on three experimental realizations for each point. The linear fit on panel (a) evidences the linear dependence of the nanometric particle generation on the input energy.

dent test providing the risk $\alpha$ that the two results are due to Gaussian sampling fluctuations. ${ }^{12}$

As shown in Fig. 1(a), the generation of nanoparticles (20-300 nm) depends almost linearly on the incident pulse energy, above a threshold of $25 \mathrm{~mJ}$ corresponding to the filamentation threshold in our conditions. This linear dependence is evidenced by the agreement $\left(R^{2}=0.95\right)$ of the linear fit (dashed curve) with the experimental data in spite of their error bars. This agreement is not further improved by considering higher-order polynomials. The energy dependence of the nanoparticles is parallel to that of the free electron release and stems from the linear scaling of the filament number, and hence of the active volume, with the incident power. ${ }^{13}$ The effect of the laser on the concentration of larger particles (250-500 nm) depends less on energy [Fig. 1(b)], consistent with the fact that the growth of these particles, which is governed by diffusion-limited condensation, is limited not only by the presence of condensation nuclei but also by the available water vapor contents in the atmosphere around them.

The laser-assisted generation of nanometric particles on the chirp does not depend on the sign of the chirp but only on the pulse duration. This dependence displays two regimes (Fig. 2). Under up to $\sim 1.5 \mathrm{ps}$, it decreases regularly parallel to the electron release. Beyond this pulse duration, the particle generation efficiency stabilizes while the electron generation continues to decrease. Submicronic particles typically exhibit the same variation, although the measurement uncertainty is larger due to lower particle densities in those size classes (Fig. 2(b)). The decoupling between the efficiencies of nanoparticle generation and ionization suggests that the initiation of the laser action relies on photodissociative processes rather than the photoionization of the molecules of the air. These photodissociative processes generate ozone and $\mathrm{NO}_{2}$ from the molecules of the air, subsequently releasing $\mathrm{HNO}_{3}$ in the atmosphere. Only seven photons are required to photodissociate $\mathrm{N}_{2}$, as compared with 11 photons to ionize it into $\mathrm{N}\left({ }^{2} \mathrm{D}^{0}\right)+\mathrm{N}\left({ }^{4} \mathrm{~S}^{0}\right) \cdot{ }^{14,15}$ Photodissociation, therefore, depends less on the intensity than photoionization, allowing its efficiency to decrease less for longer pulses. Conversely, attachment contributes to decreasing the electron density beyond a few picoseconds. Note that the ionization of the condensed particles contributes negligibly to the total charge. ${ }^{10}$

In the considered atmospheric conditions, the incident polarization has little influence, if any, on the laser-assisted condensation process (Fig. 3) within the uncertainty of the measurement: The difference between the linear and circular polarization is not statistically significant (within $\alpha \leq 0.025$ ) for any size class. As a consequence, the ratio of the effect of pulses with both polarizations lies close to unity. This result can be understood by considering that circular polarization yields less filaments, ${ }^{16,17}$ which are, however, longer and more intense, ${ }^{18}$ so that the overall efficiency is little affected.

Finally, as displayed in Fig. 4, a loosely focused beam allowing multiple filamentation yields up to 2.5 times more particles of $400 \mathrm{~nm}$ than its strongly focused counterpart. The analysis of 12 realizations of the same experiment shows that this difference is statistically significant up to $600 \mathrm{~nm}$ but vanishes for larger particles due to the small particle counts. In particular, the apparent larger yield of particles in the $2-3 \mu \mathrm{m}$ region may very well be an artifact due to sampling fluctuations.

The higher efficiency of filaments may appear surprising at first sight, considering the high nonlinearity of the processes at play and the fact that the intensity is clamped to
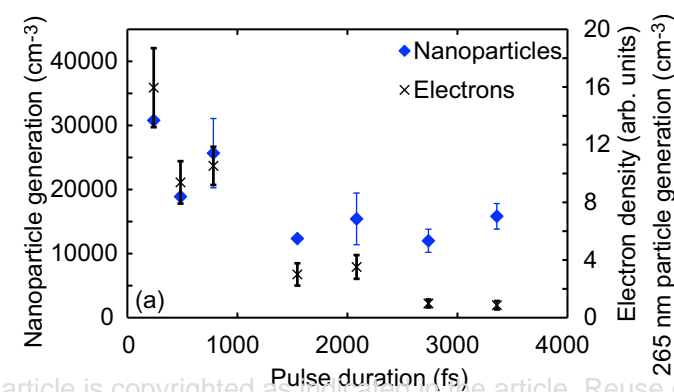

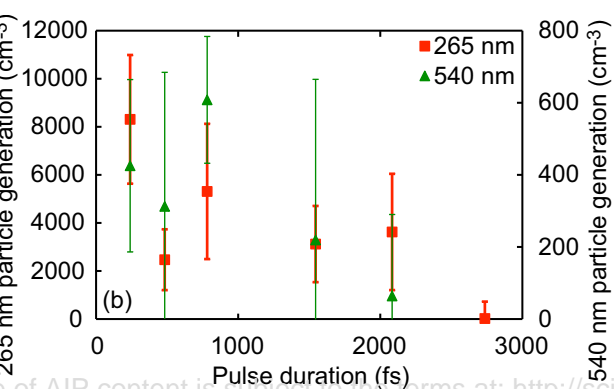

FIG. 2. (Color online) Pulse duration dependence of the generation of (a) nanometric $(25 \mathrm{~nm})$ and (b) submicrometric particles by laser pulses of 150 mJ. Atmospheric conditions are $90 \%-$ $96 \%$ relative humidity and $8{ }^{\circ} \mathrm{C}$. Error bars display the standard deviation on three measurements. 


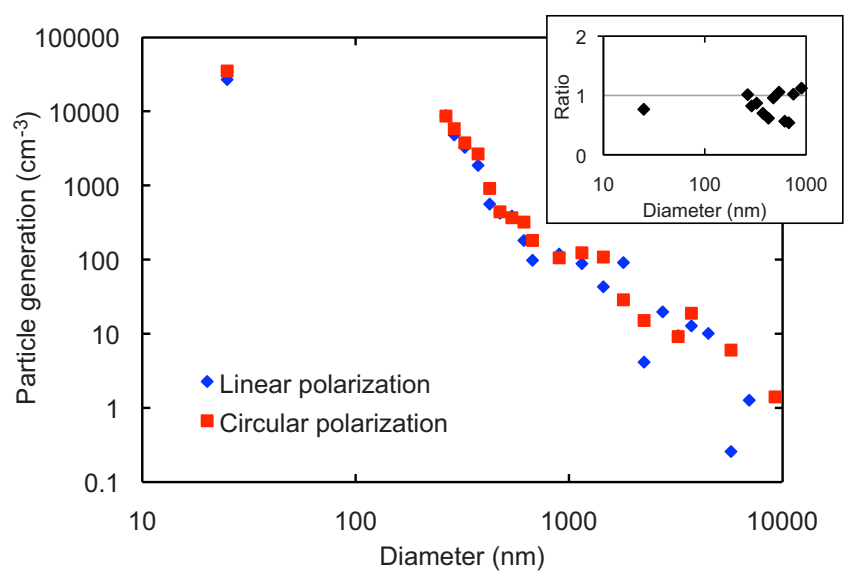

FIG. 3. (Color online) Effect of the laser filaments on the particle concentration for linear and circular incident polarizations of a $130 \mathrm{~mJ}$ and $240 \mathrm{fs}$ pulse. Atmospheric conditions are $12{ }^{\circ} \mathrm{C}$ and $80 \%$ relative humidity. Inset: Ratio of the two curves from the main panel.

$\sim 5 \times 10^{13} \mathrm{~W} / \mathrm{cm}^{2} .{ }^{19,20}$ For example, the focused beam yields three to 20 times more free electrons than its multifilamenting counterpart. However, the $\sim 10$ filaments in the latter activate a much larger volume than the strongly focused beam does. Besides the filament volume of $50 \mathrm{~mm}^{3}$, the active species like $\mathrm{HNO}_{3}$ generated by the filaments spread around them and activate the surrounding volume. This more spread volume also contains a larger amount of water vapor and is therefore more favorable to the diffusion-limited growth of the particles.

As a conclusion, after investigating laser-induced water vapor nucleation in the real atmosphere under various laser conditions, we observed that both the nucleation of nanoparticles and their growth to submicrometric sizes strongly depend on the incident energy with a linear dependence beyond

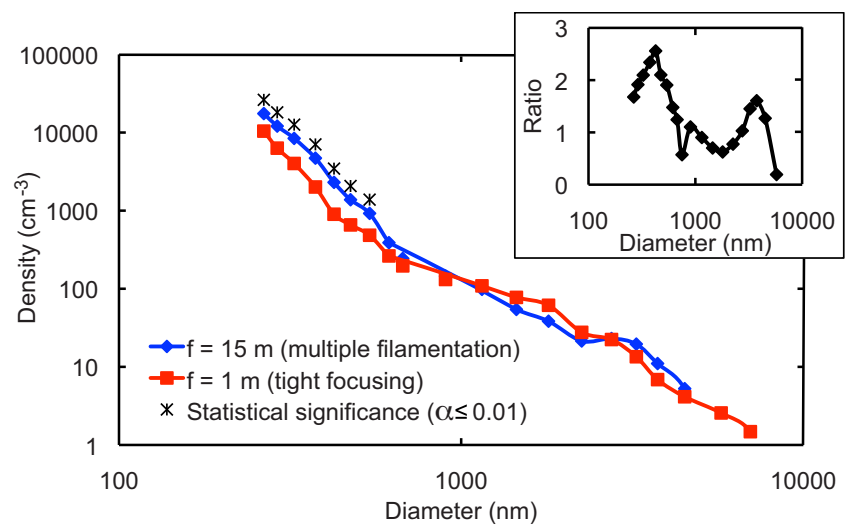

FIG. 4. (Color online) Comparison of the efficiencies of tight $(f=1 \mathrm{~m})$ and loose ( $f=15 \mathrm{~m}$, leading to multiple filamentation) laser pulses of $150 \mathrm{~mJ}$ for $240 \mathrm{fs}$. Atmospheric conditions are $66 \%$ relative humidity and $12{ }^{\circ} \mathrm{C}$. Inset: Ratio of the two curves from the main panel. the threshold of filamentation. Shorter pulses appear to be more efficient in assisting condensation, although the efficiency stabilizes above 1.5 ps. The polarization showed no influence on the efficiency, while the wider volume of a multifilamenting beam appears to be more favorable to the generation of droplets up to $600 \mathrm{~nm}$. The discrepancy between the behaviors of the particle generation and ionization suggest that the first steps of laser-assisted condensation rely more on photodissociation than on photoionization of the air molecules. These results are of key interest to dimension laser systems to optimize laser-assisted condensation.

This work was supported by the Deutsche Forschungsgemeinschaft and the Fonds National Suisse de la Recherche Scientifique (FNS Grant Nos. 200021-125315 and 200021125315-2).

${ }^{1}$ J. Kasparian, M. Rodriguez, G. Méjean, J. Yu, E. Salmon, H. Wille, R. Bourayou, S. Frey, Y.-B. André, A. Mysyrowicz, R. Sauerbrey, J.-P. Wolf, and L. Wöste, Science 301, 61 (2003).

${ }^{2}$ A. Couairon and A. Mysyrowicz, Phys. Rep. 441, 47 (2007).

${ }^{3}$ L. Bergé, S. Skupin, R. Nuter, J. Kasparian, and J.-P. Wolf, Rep. Prog. Phys. 70, 1633 (2007).

${ }^{4}$ J. Kasparian and J.-P. Wolf, Opt. Express 16, 466 (2008).

${ }^{5}$ S. L. Chin, S. A. Hosseini, W. Liu, Q. Luo, F. Théberge, N. Aközbek, A. Becker, V. P. Kandidov, O. G. Kosareva, and H. Schröder, Can. J. Phys. 83, 863 (2005).

${ }^{6}$ P. Rohwetter, J. Kasparian, K. Stelmaszczyk, Z. Hao, S. Henin, N. Lascoux, W. M. Nakaema, Y. Petit, M. Queißer, R. Salamé, E. Salmon, L. Wöste, and J.-P. Wolf, Nat. Photonics 4, 451 (2010).

${ }^{7}$ I. Langmuir, Science 106, 505 (1947).

${ }^{8}$ Y. Petit, S. Henin, J. Kasparian, and J.-P. Wolf, Appl. Phys. Lett. 97, 021108 (2010).

${ }^{9}$ H. Wille, M. Rodriguez, J. Kasparian, D. Mondelain, J. Yu, A. Mysyrowicz, R. Sauerbrey, J.-P. Wolf, and L. Wöste, Eur. Phys. J.: Appl. Phys. 20, 183 (2002).

${ }^{10}$ S. Henin, Y. Petit, D. Kiselev, J. Kasparian, and J.-P. Wolf, Appl. Phys. Lett. 95, 091107 (2009).

${ }^{11}$ J. Yu, D. Mondelain, J. Kasparian, E. Salmon, S. Geffroy, C. Favre, V. Boutou, and J.-P. Wolf, Appl. Opt. 42, 7117 (2003).

${ }^{12}$ W. H. Press, S. A. Teukolsky, W. T. Vetterling, and B. P. Flannery, Numerical Recipes in C: The Art of Scientific Computing (Cambridge University Press, Cambridge, England, 1992).

${ }^{13}$ G. Méjean, J. Kasparian, J. Yu, E. Salmon, S. Frey, J.-P. Wolf, S. Skupin, A. Vinçotte, R. Nuter, S. Champeaux, and L. Bergé, Phys. Rev. E 72, 026611 (2005).

${ }^{14}$ P. G. Richards, D. G. Torr, and M. R. Torr, J. Geophys. Res. 86, 1495 (1981)

${ }^{15}$ E. C. Zipf, P. J. Espy, and C. F. Boyle, J. Geophys. Res. 85, 687, DOI:10.1029/JA085iA02p00687 (1980).

${ }^{16}$ G. Fibich and B. Ilan, Phys. Rev. E 67, 036622 (2003).

${ }^{17}$ L. Bergé, C. Gouédard, J. Schjødt-Eriksen, and H. Ward, Physica D 176, 181 (2003).

${ }^{18}$ S. Petit, A. Talebpour, A. Proulx, and S. L. Chin, Opt. Commun. 175, 323 (2000).

${ }^{19}$ J. Kasparian, R. Sauerbrey, and S. L. Chin, Appl. Phys. B: Lasers Opt. 71, 877 (2000).

${ }^{20}$ A. Becker, N. Aközbek, K. Vijayalakshmi, E. Oral, C. M. Bowden, and S. L. Chin, Appl. Phys. B: Lasers Opt. 73, 287 (2001). 